\title{
A Novel Ontology Analysis Tool
}

\author{
Zhixiao Wang*, Shixiong Xia and Qiang Niu \\ School of Computer Science and Technology, China University of Mining and Technology, Xu Zhou, 221116, China
}

Received: 12 Jun. 2013, Revised: 18 Oct. 2013, Accepted: 20 Oct. 2013

Published online: 1 Jan. 2014

\begin{abstract}
With the development of semantic web, more and more ontologies are developed for various purposes. In order to evaluate these ontologies, there have emerged numerous ontology analysis approaches. This paper introduces the classical field theory to ontology area, and puts forward a semantic field model to analyze ontology characteristics from the perspective of concept interaction and hierarchical clustering. In semantic field, the equipotential lines present a natural nesting structure. The equipotential lines' distribution corresponds with the semantics' distribution resulting from the interaction and association of ontology concepts. Based on equipotential line distribution, we can analyze concept hierarchical clustering characteristics. Experiments showed that the semantic field does well in ontology concept hierarchical clustering analysis. The semantic field model has good scalability, and clustering execution time follows an approximate linear relationship with concept scale.
\end{abstract}

Keywords: Ontology; Semantic Field; Concept Hierarchical Clustering.

\section{Introduction}

Ontologies provide a commonly agreed understanding about domain knowledge, and as such, are becoming a crucial element of the Semantic Web. Although many definitions have been given for the term ontology, the most common and simply stated is that ontology is a 'specification of a shared conceptualization' [1]. An ontology specifies a shared domain vocabulary to describe concepts, their properties and relations.

In order to evaluate these ontologies, there have emerged numerous ontology analysis approaches [2]. Some of them evaluate ontologies for correctness, completeness and redundancy; some of them are used to ensure the consistency of ontology throughout its entire lifetime; more recent approaches are proposed to analyze the reuse and adaptation of existing ontologies.

This paper puts forward a novel analysis method named semantic field. Different from above approaches, the semantic field analyzes ontology characteristic from the perspective of concept interaction and hierarchical clustering. The semantic field model derives from classical field theory. The latter was used to describe non-contact interaction between matter particles. Later on, people abstracted it as a mathematical model to describe non-contact interaction between objects, and to depict distribution law of physical quantities. In ontology, concepts are not isolated. There exist non-contact semantic interaction and association among them. Table 1 shows the analogies between electrostatic field and semantic field. In this light, we introduce classical field theory to ontology area, and put forward semantic field model.

Table 1: Analogies between electrostatic field and semantic field

\begin{tabular}{cc}
\hline electrostatic field & semantic field \\
\hline electron & ontology concept \\
electron energy & importance of concept \\
electrostatic force & concept interaction \\
\hline
\end{tabular}

In semantic field, the equipotential lines present a natural nesting structure. The equipotential lines' distribution corresponds with the semantics' distribution resulting from the interaction and association of ontology concepts. Based on equipotential line distribution, we can reveal concept hierarchical clustering characteristics within an ontology. The semantic field can show which concepts are located in clustering center, and have powerful influence on surrounding concepts. These are important concepts for the ontology, and should be paid enough attention to in various ontology operations.

\footnotetext{
*Corresponding author e-mail: zhxwang@cumt.edu.cn
} 
Except for analyzing concept interaction and hierarchical clustering within an ontology, the semantic filed can also be used in semantic-based resource clustering, resource organization and search in $\mathrm{P} 2 \mathrm{P}$, etc. These semantic field applications are not the research scope of this paper. More details can be referred to our previous work [3].

\section{Related Works}

Faraday put forward field concept in area of thermophysics and electromagnetics. Later on, people abstracted it as a mathematical model to describe non-contact interaction between objects. The concept of semantic field has been hotly debated in linguistic community, which was used to analyze semantic relations between vocabularies in glossary system [4]. To the best of our knowledge, there are a few literatures about this topic in computer science and information technology area.

Ismael Navas-Delgado et al [5] presented the Semantic Field which enables the global comparison of ontologies. The semantic field uses the results obtained from ontology matching tools to estimate the global similarity between ontologies. It can discover the global configuration of relationships between existing ontologies, and had been used in a tool called the Semantic Field Tool (SemFiT). They also published other research literature about this topic in [6]. The semantic field in [5] and [6] is a set of relevant ontologies.

Kun Yue and Weiyi Liu [7], inspired by the theories of lexical semantics and electrostatic field, proposed a theoretical model called semantic field. In the method, text documents are regarded as semantic packages and words are regarded as semantic elements covering these packages. The semantic filed was used to reflect the similarity or dissimilarity between semantic elements or semantic packages in information retrieval systems. They took automatic keyword extraction as the representative application of the proposed semantic field. The semantic field in [7] takes semantic elements and semantic packages in text documents as research target.

Benjamin Stone and Simon Dennis [8,9] outlined a method named semantic field for estimating the visual saliency different areas displayed on a web page. Latent Semantic Analysis is used to calculate Semantic Fields values of any $(\mathrm{x}, \mathrm{y})$ coordinate point on a web page based on the structure of that web page. These Semantic Field values were then used to predict eye-tracking data. The semantic field in [8] and [9] is based on four semantic field model: word overlap, Vector Space Model, Latent Semantic Analysis, and Sparse Nonnegative Matrix Factorization.

Different from above works, this paper introduces the classical field theory to ontology area, and puts forward a semantic field model to analyze ontology characteristic from the perspective of concept interaction and hierarchical clustering.

\section{Semantic Field Model}

\subsection{Semantic field construction}

Definition 1.Given ontology $O$ and its concept set $C=\left\{c_{1}, c_{2}, \ldots, c_{i}, \ldots, c_{n}\right\}$. The representative concept set (obtained by using complex network technology [10]) is $C^{\prime}=\left\{c_{1}^{\prime}, c_{2}^{\prime}, \ldots, c_{i}^{\prime}, \ldots, c_{m}^{\prime}\right\}, \mathrm{m}<$ n.Constructing $\quad \mathrm{a}$ m-dimensional space $R^{\mathrm{m}}$, the i-th dimension of $R^{\mathrm{m}}$ is semantic similarity between representative concept $C_{i}^{\prime}$ and all ontology concepts $(1 \leq i \leq m) . R^{\mathrm{m}}$ is called the semantic space of ontology $O$, denoted by $\Omega_{o}$.

Once semantic space $\Omega_{o}$ is established, all concepts of ontology $O$ can be put into $\Omega_{o}$.

Definition 2. Given ontology $O$ and its corresponding semantic space $\Omega_{o}$, the position vector of concept $c_{i}$ in $\Omega_{o}$ is $x_{c i}=\left\{s_{i 1}, s_{i 2}, \ldots, s_{i k}, \ldots, s_{i m}\right\}$, the k-th dimension $s_{i k}$ is:

$$
s_{i k}=\operatorname{sim}\left(c_{i}, c_{k}^{\prime}\right) \quad 1 \leq i \leq n, 1 \leq k \leq m
$$

where, $c_{k}^{\prime}$ is representative concept of ontology $O ; \operatorname{sim}\left(c_{i}\right.$, $c_{k}^{\prime}$ ) is the semantic similarity between $c_{i}$ and $c_{k}^{\prime}$.

In this paper, we use semantic similarity measure proposed by $\mathrm{Li}$ et al [11], which significantly outperformed traditional similarity measures [12].

$$
\sin \left(c_{i}, c_{k}^{\prime}\right)=\left\{\begin{array}{cc}
e^{-\alpha \mathrm{l}} \cdot \frac{e^{\beta \mathrm{h}}-e^{-\beta \mathrm{h}}}{e^{\beta \mathrm{h}}+e^{-\beta \mathrm{h}}}, & c_{i} \neq c_{k}^{\prime} \\
1, & \text { otherwise }
\end{array}\right.
$$

where, $l$ is the shortest path length between concept $c_{i}$ and concept $c_{j}, h$ is the depth of the lowerest common ancestor in the ontology. $\alpha$ and $\beta$ are parameters scaling the contribution of shortest path length and depth, respectively. The optimal values are as follow: $\alpha=0.2$, $\beta=0.6$.

Definition 3.Given ontology $O$ and its corresponding semantic space $\Omega_{o}$. All ontology concepts are mapped into $\Omega_{o}$. Ontology concepts are regarded as field sources, and these concepts interact and associate with each other, forming one field called semantic field.

Semantic field depicts interaction among ontology concepts and semantic distribution law within an ontology. We can describe semantic field from the perspectives of potential, gradient and intensity. Potential represents the semantics of a certain position; gradient describes local semantics change of semantic field; and field intensity reflects the strength of semantics association among concepts.

Research showed that the influence scope of one field source is limited in short-range field, and potential value will quickly attenuate to zero with distance increasing. In semantic field, the influence scope of semantic 
association among concepts is limited, too. That is to say: semantic field presents properties of short-range field to some extent. The typical potential function of short-range field is Gaussian function, so this paper uses it as the potential function of semantic field.

Definition 4.Given concept (field source) set $C=\left\{c_{1}\right.$, $\left.c_{2}, \ldots, c_{i}, \ldots, c_{n}\right\}$ of ontology $O$ and its corresponding position set $P=\left\{x_{c 1}, x_{c 2}, \ldots, x_{c i}, \ldots, x_{c n}\right\}$ in $\Omega_{o} . x_{c i}$ is position vector of concept $c_{i}(1 \leq i \leq n)$. The potential of any field position $x$ is defined as:

$$
\varphi(x)=\sum_{i=1}^{n} \varphi_{c i}(x)=\sum_{i=1}^{n}\left(m_{c i} \cdot e^{-\left(\frac{\left\|\mathrm{x}_{\mathrm{ci}}-\mathrm{x}\right\|}{\sigma}\right)^{2}}\right)
$$

where, $n$ is ontology concept number; $\varphi_{c i}(x)$ is potential produced by concept $c_{i}$ at $x ; m_{c i}$ is concept mass of $c_{i}$ , $m_{c i} \geq 0 ; \sigma$ is influence factor, $\sigma \in(0,+\infty) ;\left\|x_{c i}-x\right\|$ is semantic distance between $x_{c i}$ and $x$. If the $\left\|x_{c i}-x\right\|$ exceeds the influence scope of $c_{i}$, then $\varphi_{c i}(x)=0$.

In fact, field intensity function $F(x)$ and potential function $\varphi(x)$ is equivalent. They are connected together by differential operator $\nabla, F(x)=\nabla \varphi(x)$. Therefore, we can get field intensity function $F(x)$ via the differential of potential function $\varphi(x)$. Here, we no longer describe intensity definition and gradient definition in detail.

\subsection{Ontology concept mass calculation}

Potential computation involves ontology concept mass $m$. Apparently, matter particle has its inherent mass. But how to weigh ontology concept mass? Concept mass should reflect the importance of the concept. The more important the concept is, the bigger the mass is. In this paper, we adapt PageRank [13] algorithm, which is widely used in search engine area, to calculate ontology concept mass.

The PageRank algorithm was put forward by Sergey Brin and Larry Page, which had been applied successfully in google search engine. The PageRank assumes that a web page's importance is measured by the number and importance of other web pages linking to the page. Similarly, the concept importance can be measured by the number and importance of its sub-concepts. The more sub-concepts one concept has, the more important the concept is. The contributions of these sub-concepts are different: the more important the sub-concept itself is, the more contribution the sub-concept makes to the concept.

Definition 5. Given ontology $O$ and its concept set $C=$ $\left\{c_{1}, c_{2}, \ldots, c_{i}, \ldots, c_{n}\right\}$. The mass of concept $\mathrm{A}$ is defined as follows:

$$
m(A)=(1-d)+d \cdot\left(\frac{m\left(c_{1}\right)}{s\left(c_{1}\right)}+\frac{m\left(c_{2}\right)}{s\left(c_{2}\right)}+\ldots+\frac{m\left(c_{k}\right)}{s\left(c_{k}\right)}\right)
$$

where, $m(A)$ is mass of concept $\mathrm{A} ; c_{1}, c_{2}, \ldots, c_{k}$ are sub-concepts of concept $\mathrm{A} ; m\left(c_{i}\right)$ is concept mass of $c_{i}(i=1,2, \ldots k) ; s\left(c_{i}\right)$ is parent concept number of $c_{i}$, normally 1 ; $d$ is damping factor, $0<d<1$, generally set at 0.85 .

\subsection{Influence factor selection}

The influence factor $\sigma$ will affect semantic field. If $\sigma$ is small, the semantic association among concepts is weak. And when $\sigma \rightarrow 0$, there is even no semantic association among concepts. Conversely, if $\sigma$ is big, the semantic association becomes strong, and in extreme condition all ontology concepts associates with each. Therefore, we need select suitable $\sigma$ value, so as to make the semantic field distribution reflect the real internal distribution of semantics as much as possible.

This paper adopt potential entropy [14] to evaluate the rationality of potential field distribution. In information theory, the shannon entropy reflects system uncertainty. The bigger the shannon entropy is, the more uncertain the system is; Conversely, the smaller it is, the least uncertain the system is. When field achieves the smallest shannon entropy, the influence factor value is optimal.

Suppose the potential values of concepts $c_{1}, c_{2}, \ldots, c_{n}$ are $\varphi_{c 1}, \varphi_{c 2}, \ldots, \varphi_{c n}$ respectively, the potential entropy $\mathrm{H}$ is defined as:

$$
H=-\sum_{i=1}^{n} \frac{\varphi_{c i}}{Z} \cdot \log \left(\frac{\varphi_{c i}}{Z}\right)
$$

where, $n$ is ontology concept number; $Z=\sum_{i=1}^{n} \varphi_{c i}$ is a normalization factor. In essence, influence factor selection is the problem of function $H(\sigma)$ minimization. That is:

$$
\min H(\sigma)=\min -\sum_{i=1}^{n} \frac{\varphi_{c i}}{Z} \cdot \log \left(\frac{\varphi_{c i}}{Z}\right)
$$

This paper adopts golden section method [15] to solve this problem, and the algorithm is described as follow.

Algorithm 1. Influence factor selection algorithm

Input: ontology concept set $C=\left\{c_{1}, c_{2}, \ldots, c_{i}, \ldots, c_{n}\right\}$, position set $P=\left\{x_{c 1}, x_{c 2}, \ldots, x_{c i}, \ldots, x_{c n}\right\}$, degree of accuracy $\varepsilon$

Output: optimal influence factor $\sigma$

Description:

$$
\begin{aligned}
& a=\frac{\sqrt{2}}{3} \min _{c i \neq c j}\left\|x_{c i}-x_{c j}\right\|, b=\frac{\sqrt{2}}{3} \max _{c i \neq c j}\left\|x_{c i}-x_{c j}\right\| \\
& \sigma_{M}=a+(1-\tau)(b-a), \sigma_{N}=a+\tau(b-a), \tau=\frac{-1+\sqrt{5}}{2} \\
& \text { Calculating } H_{M}=H\left(\sigma_{M}\right) \text { and } H_{N}=H\left(\sigma_{N}\right) \\
& \text { While }|b-a|>\varepsilon \text { do } \\
& \text { If } H_{M}<H_{N} \text { then } \\
& \qquad b=\sigma_{N}, \sigma_{N}=\sigma_{M}, \\
& \quad \sigma_{M}=a+(1-\tau)(b-a), H_{N}=H_{M} \\
& \text { Calculating } H_{M}=H\left(\sigma_{M}\right) \\
& \text { ELSE } \\
& \qquad a=\sigma_{M}, \sigma_{M}=\sigma_{N}, \\
& \quad \sigma_{N}=a+\tau(b-a), H_{M}=H_{N}
\end{aligned}
$$


End While

Calculating $H_{N}=H\left(\sigma_{N}\right)$

If $H_{M}<H_{N}$ then $\sigma=\sigma_{M}$

Else $\sigma=\sigma_{N}$

Return $\sigma$

\section{Semantic Field Based Concept Hierarchical Clustering}

In semantic field, if connect the positions with same potential value, we can get a series of equipotential lines. These equipotential lines present a natural nesting structure. The equipotential lines' distribution corresponds with the semantics' distribution resulting from the interaction and association of ontology concepts in semantic space. Based on equipotential line distribution, we can discover the hierarchical clustering characteristics of ontology concepts.

\subsection{Initial clustering center selection}

Potential reflects influence capability of ontology concept in whole semantic space. Potential heart is local maximum position in semantic field, which can be regarded as virtual field source. Under the attraction of these virtual field sources, ontology concepts in semantic filed show self-organizing clustering characteristic. In the semantic field formed by single concept, potential heart is the position where the concept itself lying. For the semantic field formed by multiple concepts, we can search all potential heart positions along the direction of gradient by using climbing method.

If position $x$ is potential heart, for any position $y, x$ should satisfy the following formula:

$$
\forall y \wedge y \in \text { neighbor }(x) \rightarrow \varphi(x) \geq \varphi(y)
$$

where, neighbor $(x)$ are surrounding positions of $x, \varphi(x)$ is potential value of position $x, \varphi(y)$ is potential value of position $y$.

Figure 1 is parts of SWRC [16] ontology concepts, Figure 2 shows the semantic field in two-dimensional semantic space formed by these concepts, and potential hearts are obvious.

Because of potential superposition, potential heart does not coincide with ontology concept. We select the concept being closest to the potential heart as initial clustering center. The potential heart number determines the initial clustering number.

\section{2. hierarchical clustering algorithm description}

Iteratively merging initial clustering center based on saddle points between two local maximum positions [14], we can get hierarchical clustering of ontology concepts.

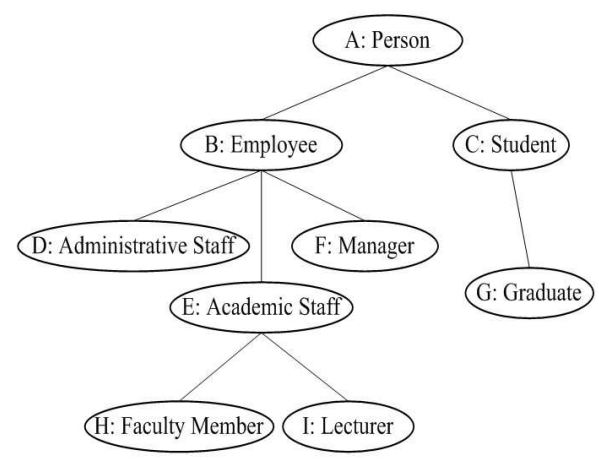

Figure 1: Parts of SWRC ontology concepts

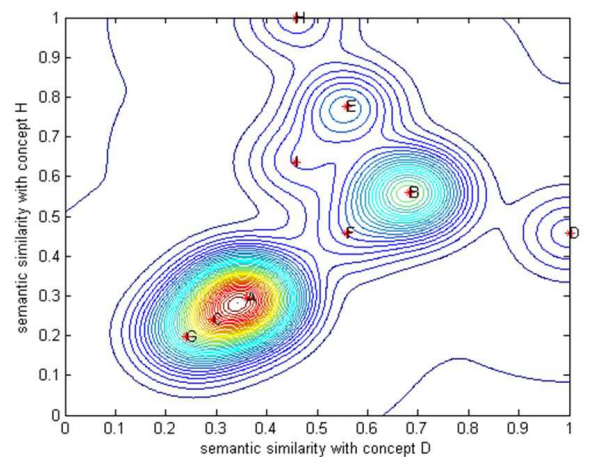

Figure 2: Semantic field in two-dimensional semantic space ( $\sigma=$ $0.1)$

Algorithm 2. semantic field based concept hierarchical clustering algorithm

Input: ontology concept set $C=\left\{c_{1}, c_{2}, \ldots, c_{i}, \ldots, c_{n}\right\}$ ,position set $P=\left\{x_{c 1}, x_{c 2}, \ldots, x_{c i}, \ldots, x_{c n}\right\}$, noise threshold $\varepsilon$ $\left.C L_{k}\right\}$

Output: concepts hierarchical division $\left\{C L_{0}, C L_{1}, \ldots\right.$,

\section{Description:}

(1) select optimal influence factor $\sigma$ using algorithm 1;

(2) $M a p=$ CreateMap $(C, \sigma)$; // Mesh generation and index tree construction

(3) CriticalPoints = Search_CriticalPoints $($ Map, $\sigma)$; //Searching topology critical points

(4) MaxPoints is local maximum position set, SadPoints is saddle points set;

(5) $C L_{0}=$ Initiallization_Partition(Map,C,Max Points, $\sigma, \varepsilon)$; //Ontology concepts initial partition based on local maximum positions;

(6) $\left\{C L_{0}, C L_{1}, \ldots, C L_{k}\right\}=$ Partition_Merge(Map, $C L_{0}$, MaxPoints, SadPoints, $\left.\sigma, \varepsilon\right)$; // Iteratively merging initial partition based on saddle points between two local maximum positions;

Figure 3 shows the hierarchical clustering results of ontology concepts in Figure 1 based on algorithm 2. 


\section{Experiments and Results}

The goal of experiments is to investigate the effectiveness of semantic field as an ontology analysis tool. Simulation program implemented with java and relative tools in the Linux environment. All ontologies used in experiments come from Protégé Ontology Library of Stanford University

(http://protegewiki.stanford.edu/wiki/Protege_Ontology_Library).

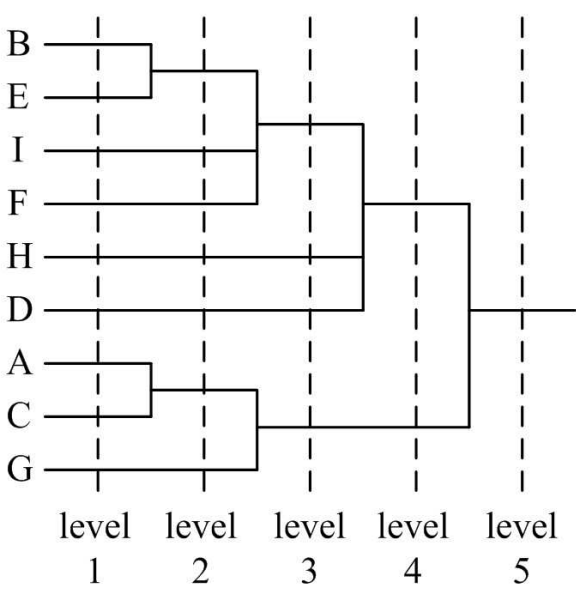

Figure 3: ontology concepts clustering spectra

\subsection{Clustering error rate}

We selected three ontologies: ka ontology (an ontology about concepts from academic research), hu ontology (a hierarchic division of hydrologic units), and finance ontology (an ontology on financial instruments, involved parties, processes and procedures in securtities handling). We carried out semantic field based concept hierarchical clustering for each ontology, and then compared these results with manual results done by domain experts. This paper defines an evaluation criterion named clustering error rate as follows:

$$
\frac{\text { concept number of clustering error }}{\text { total concept number }}
$$

Figure 4 shows the clustering error rate of three ontologies at level 2, level 3 and level 4.

On the whole, the semantic field does well in ontology concept hierarchical clustering. The more the clustering partition, the higher the clustering error rate; on the contrary, the less the clustering partition, the lower the clustering error rate. Experiment also shows that ontology structure may affect the hierarchical clustering performance. Comprehensive ontology structure can

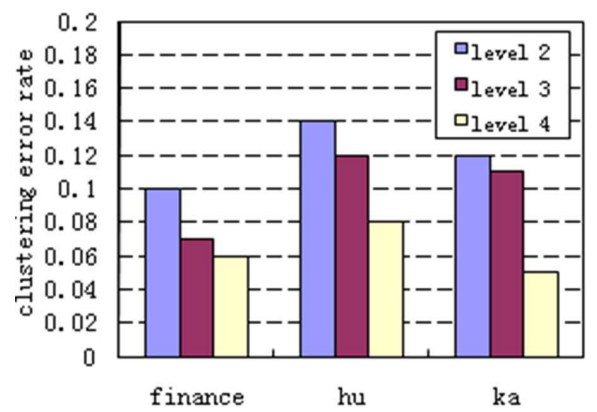

Figure 4: Clustering error rate

better reveal concept clustering characteristics. hu ontology is a simple hierarchic division of hydrologic units, therefore, the clustering error rate is relatively high.

\subsection{Scalability}

We selected 20 ontologies from Protégé Ontology Library to evaluate the scalability of this method. Ontology concept scale range from 4633 to 102 . Figure 5 shows the execution time of 20 ontologies. The axis is concept number of each ontology, and the axis is clustering execution time of each ontology. The result shows that this method has good scalability. Concept clustering execution time follows an approximate linear relationship with concept scale.

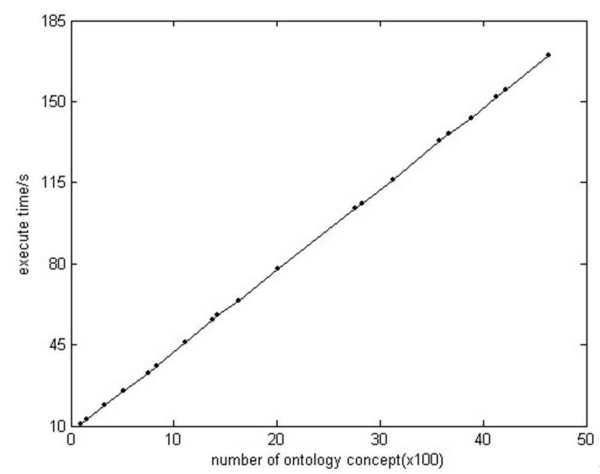

Figure 5: Scalability of semantic field based concept hierarchical clustering

\subsection{Influence of ontology evolution}

Ontology is not static. Ontology evolution means modifying or upgrading the ontology when there is a certain need for change or there comes a change in the 
domain knowledge [17]. Ontology evolution generally includes concept addition, concept deletion, and concept translocation. We carried out experiment to evaluate the influence of ontology evolution on hierarchical clustering results.

In Figure1, supposes the leaf concept "F: Manager" is deleted. Figure 6 shows the semantic field in two-dimensional semantic space after concept deletion. The hierarchical clustering results of other concepts does not changed after leaf concept "F: Manager" deleted.

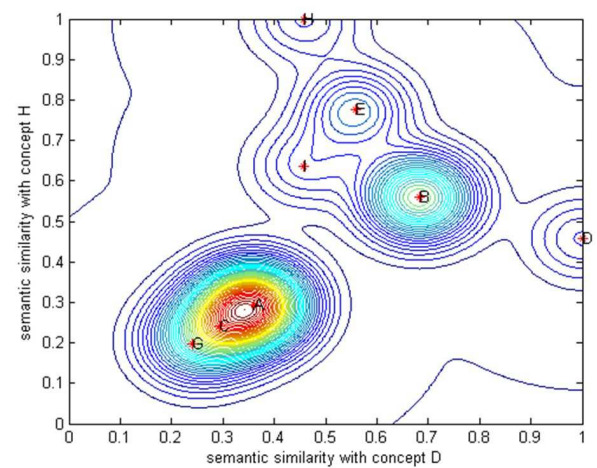

Figure 6: Semantic field after concept deletion $(\sigma=0.1)$

In Figure 1, supposes a leaf concept "J: Assistant Professor" is added the sub-concept of concept "H: Faculty Member". Figure 7 shows the semantic field in two-dimensional semantic space after concept addition. The hierarchical clustering results of other concepts does not change after leaf concept "J: Assistant Professor" added.

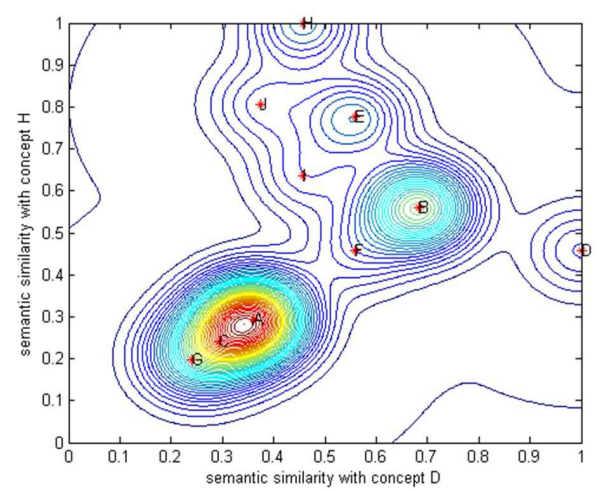

Figure 7: Semantic field after concept addition $(\sigma=0.1)$

Figure 6 and Figure 7 showed that the influence of ontology evolution on semantic field is local. Ontology evolution does not change the hierarchical clustering results of other concepts.

\section{Conclusion}

Ontologies are becoming a crucial element of the Semantic Web. In order to evaluate these ontologies, there have emerged numerous ontology analysis approaches. This paper introduces the classical field theory to ontology area, and puts forward a semantic field model to analysis ontology characteristics from the perspective of concept interaction and hierarchical clustering. In semantic field, the equipotential lines present a natural nesting structure. The equipotential lines' distribution corresponds with the semantics' distribution resulting from the interaction and association of ontology concepts. Based on equipotential line distribution, we can analyze concept hierarchical clustering characteristic.

The semantic field based hierarchical clustering can show which concepts are located in clustering center, and have powerful influence on surrounding concepts. These are important concepts for the ontology. We should pay enough attention to these concepts in various ontology operations.

\section{Acknowledgements}

This work was supported by the Fundamental Research Funds for the Central Universities No.2013XK10.

Our sincere thanks go to Professor Dalu Zhang, the doctoral supervisor of first author Zhixiao Wang, who introduces field theory to us to solve scientific problems.

\section{References}

[1] Gruber CTR. A translation approach to portable ontologies. Knowledge Acquisition, 5,199-220 (1993).

[2] Valerie Cross, Anindita Pal. An ontology analysis tool. International Journal of General Systems, 37, 17-44 (2008).

[3] Zhixiao Wang, Dalu Zhang, Guangjie Yu, et al. Semantic field model. Journal of Tongji University (nature science), 37, 1526-1530 (2009).

[4] Marta Carretero. The Semantic Field of Modal Certainty: A Corpus-Based Study of English Adverbs. Journal of Pragmatics, 40, 1640-1644 (2008).

[5] Ismael Navas-Delgado, Maria del Mar Roldn-Garcła, Jos F. Aldana-Montes. Semantic fields: finding ontology relationships. LNCS, 5690, 427-434 (2009).

[6] I. Navas, I. Sanz, J.F. Aldana, R. Berlanga. Automatic generation of semantic fields for resource discovery in the semantic web. LNCS, 3588, 706-715 (2005).

[7] Kun Yue, Weiyi Liu. Semantic field: a theoretical perspective of modeling information retrieval. International journal on artificial intelligence tools, 18, 825-851 (2009).

[8] Stone, B., Dennis, S.. Using LSA Semantic Fields to predict eye movement on web pages. Proceedings of the twenty ninth conference of the cognitive science society. Lawrence Erlbaum Associates, Mahwah, NJ, 665-670 (2007). 
[9] Benjamin Stone, Simon Dennis. Semantic models and corpora choice when using Semantic Fields to predict eye movement on web pages. International Journal of HumanComputer Studies, 69, 720-740 (2011).

[10] Dalu Zhang, Zhixiao Wang, Wen Liu, et al. Ontology structure analysis based on complex network. Journal of Tongji University (nature science), 37, 258-261 (2009).

[11] Li Yuhua, Bandar Zuhair A, McLean David. An approach for measuring semantic similarity between words using multiple information sources. IEEE Trans. on Knowledge and Data Engineering, 15, 871-882 (2003).

[12] Angelos Hliaoutakis, Giannis Varelas, Euripides G.M. Petrakis, et al. MedSearch: A Retrieval System for Medical Information Based on Semantic Similarity. 10th European Conference on Research and Advanced Technology for Digital Libraries. Alicante, Spain. September, 17-22, 512515 (2006).

[13] Sergey Brin , Lawrence Page. The anatomy of a large-scale hypertextual web search engine. Computer networks and ISDN systems, 30, 107-117 (1998)

[14] Li DY, Du Y. Artificial Intelligence with Uncertainty. Beijing: National Defense Industry Press, 187-217 (2005).

[15] Jianjun Huang, Xiongwei Zhang, Yafei Zhang, et al. A novel speech enhancement algorithm based on data field. Signal Processing, 27, 1200-1205 (2011).

[16] Sure Y, Bloehdorn S, Haase P, et al. The swrc ontology-semantic web for research communities. Proceedings of 12th Portuguese Conference on Artificial Intelligence,LNCS, 3803, 218-231 (2005).

[17] Haridimos Kondylakis, Dimitris Plexousakis. Ontology evolution in data integration: query rewriting to the rescue. LNCS, 6998, 393-401 (2011).

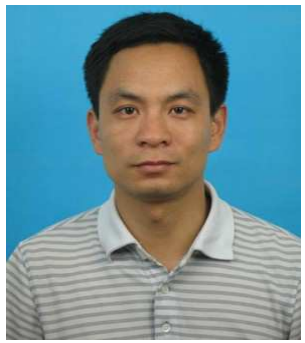

Zhixiao Wang is an associate professor of College of Computer Science and Technology, China University of Mining and Technology. $\mathrm{He}$ received his $\mathrm{PhD}$ degree in the Department of Computer Science and Engineering at Tongji Universtiy in 2011, and the BSc and MSc degrees in computer science from China University of Mining and Technology, in 2001 and 2004, respectively;His research interests include field theory application, information retrieval and data mining. He has published more than 10 peer-reviewed research papers in journals and international conferences.

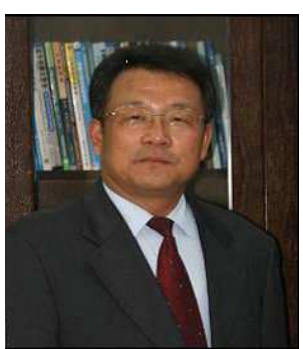

Shi Xiong Xia is born in 1962, Ph.D. He is a professor at school of Computer Science and Technology in CUMT. He has published more than 60 research papers in journals and international conferences. His research interest is Wireless sensor networks and intelligent information processing et al.

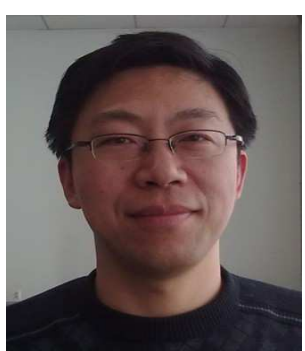

Qiang Niu is born in 1974, doctor, an associate professor in China University of Mining and Technology, received B.S. in Northeastern University in 1997, received Master in 2004 and PHD in 2010 in China University of Mining and Technology. His main research interests algorithms and data mining. are intelligent optimization 